\title{
Exculpation and Stigma in Tourette Syndrome
}

\section{An Experimental Philosophy Study}

\author{
Jo Bervoets (1) - Jarl K. Kampen (1D) • \\ Kristien Hens
}

Received: 26 March 2021 / Accepted: 27 October 2021 / Published online: 3 February 2022

(C) The Author(s) 2022

\begin{abstract}
Purpose There is a widespread recognition that biomedical explanations offer benefits to those diagnosed with a mental disorder. Recent research points out that such explanations may nevertheless have stigmatizing effects. In this study, this 'mixed blessing' [2] account of biomedical explanations is investigated in a case of philosophical interest: Tourette Syndrome.

Method We conducted a vignette survey with 221 participants in which we first assessed quantitative attributions of blame as well as the desire for social distance for behavior associated with Tourette Syndrome.
\end{abstract}

J. Bervoets $(\varangle) \cdot$ K. Hens

Department of Philosophy, NeuroEpigenEthics, University of Antwerp, Antwerp, Belgium

e-mail: joberv@gmail.com

K. Hens

e-mail: kristien.hens@uantwerpen.be

\section{J. K. Kampen}

Biometris, Wageningen University \& Research Center,

Wageningen, The Netherlands

e-mail: jarl.kampen@uantwerpen.be

J. K. Kampen

Department of Epidemiology and Medical Statistics/

StatUa, University of Antwerp, Antwerp, Belgium

K. Hens

Centre for Logic and Philosophy of Science, Higher Institute of Philosophy, Katholieke Universiteit Leuven, Leuven, Belgium
Results In our study, it is confirmed that in the case of biomedical explanations, less blame is attributed than in the case of psychosocial explanations. When presented with a mixed (or an epigenetic) explanation stressing an entanglement of biological and psychosocial factors this did not increase blame attribution. The desire for social distance is unaffected by the type of explanation but the participants' free text feedback indicates this might obfuscate an underlying dilemma between stigma and blame revealed in recent research.

Conclusion There seems to be potential for blame reduction in explanations where biological and psychosocial factors are entangled. However, dynamic, 'epigenetic', explanations require further qualitative research to be performed as well as a philosophical framework to account for the 'mixed blessings' account.

Keywords Tourette syndrome $\cdot$ Stigma .

Exculpation $\cdot$ Experimental philosophy $\cdot$ Strawson

\section{Introduction}

Biological accounts of psychopathology have been positively received. Attribution theory, a leading theory in social psychology [1], predicts that the less responsibility one attributes to an agent, the less they will be blamed and with this, the less stigma is attached to their condition. Recent empirical studies question this prediction. They connect biomedical accounts to an increase in 
stigma. While describing psychopathologies as neurobiological indeed seems to reduce the amount of blame one receives for one's behavior, they also seem to automatically increase perceptions of a person's "dangerousness", unpredictability and prognostic pessimism. Two recent reviews of this empirical research then qualify biological accounts of psychopathology as a mixed blessing [2] or a double edged sword [3]. Their hypothesis is that biomedical models engender an essentialism with respect to psychopathologies. In so doing, the potential for exculpation of biological explanations is inherently linked to an increase in dehumanization of the subjects so diagnosed. A potential net effect of this is the increase of stigmatization and, therefore, of social exclusion. It is worth noting the relative breadth that the studies in each of these reviews span: depression, ADHD, schizophrenia, obesitas, addiction are just a subset of the issues investigated under this experimental paradigm. The findings in these studies seem to be generalizable across conditions. The investigation of specific conditions may shed further light on more general underlying phenomena related to blame and stigma.

Tourette Syndrome is a case in point of the link between neurobiological explanations and a decrease in blame attribution. As Kushner [4] explains in his history of the etiology of Tourette Syndrome, there was an intimate relationship between progressively abandoning psychogenic explanations of Tourette's ${ }^{1}$ in favor of neurobiological explanations and the positive reception of the latter for their exculpatory potential. The DSM-5 [5] describes the diagnostic criteria of Tourette Syndrome as follows: multiple motor tics and at least one phonic tic, with an onset before 18 years of age. Common examples of motor tics include eye-blinking and head-nodding; examples of phonic tics include sniffing or throat-scraping. Both the frequency and type of tics vary with environmental factors which are induced by, at least, stress, different everyday activities and spaces with related sensory load [6]. Timing of diagnosis and prognosis vary widely. Whereas in most cases onset

\footnotetext{
${ }^{1}$ We will use the official DSM-5 [5] designation of Tourette Syndrome interchangeably with that of Tourette's. The former designation is the one technically used in psychiatric contexts while the latter is mostly preferred by those so diagnosed in everyday contexts. Out of respect for this preference we will use 'Tourette's' whenever there is no direct reference to a medical context.
}

is typically estimated between 4 and 6 years of age, the diagnosis can follow much later-or not at all, e.g., because it is not sought [7]. Prognosis in many cases is good with its symptoms remitting into young adulthood [8]. Although Tourette's is often thought to be rare and tied to coprolalia (the compulsive uttering of swear words), a recent population study suggests a prevalence of around $1 \%$ of the general population, with the specific coprolalia symptom being rather $\operatorname{rare}^{2}(<15 \%)$ in the diagnosed population [10]. Whilst coprolalia is relatively rare, a tendency to nonobscene socially inappropriate behavior is relatively common [11].

This makes Tourette's a paradigmatic example for investigating the relation between biological explanations, exculpation and stigma in the current context. Indeed, as tics tend to overlap with acts normally considered blameworthy, it has been used by Schroeder [13] as the paradigmatic case of (absence of) moral responsibility based on it being a purely neurological condition $^{3}$. Schroeder constructs a philosophical argument opposing two cases, "Sarah" and "Dale" exhibiting the very same behavior. The pivotal element in assessing blameworthiness of the two individuals is the explanation of this behavior. For "Sarah" this explanation is based on construing her Tourette's as a purely neurological condition ('deficit of inhibition' located in the basal ganglia). The behavior of "Dale", on the other hand, is construed as caused by purely psychosocial elements, notably as a bad habit stemming from his upbringing. As one intuitively would consider "Dale" - but not "Sarah" - blameworthy for their behavior, this can, per Schroeder's argument, only be on account of the specific neurobiological element which is specific to the "Sarah" case. In philosophy the same assumption was made as in psychology, namely, that biological accounts, by putting certain

\footnotetext{
${ }^{2}$ Mischaracterization of Tourette Syndrome in popular culture is known to stigmatize people diagnosed, see for instance Ben Ezra and colleagues [9]. In this paper we only give the nuances necessary for the argument but we encourage readers to inform themselves as stigmatization is undoubtedly the major contributor to (at least) secondary mental health issues in the diagnosed population [12]. See also Appendix 2 of in which we give the debriefing statement of our study that was designed to at least counteract stereotypes with our participants.

3 See also Arpaly and Schroeder [14] for elaborating this philosophical interpretation of Tourette's as a part of their comprehensive moral psychology framework on desire.
} 
behavior out of the control of the individual's agency, confer an intuitive benefit as regards those individuals not being blamed for certain ranges of behavior tied to their diagnosis. This benefit is mirrored in the historical account of Tourette Syndrome as one of the reasons why neurological etiology of Tourette's is preferred in favor of the psychogenic one [4].

As it was described above for psychopathological conditions in general, also for Tourette Syndrome, there is empirical evidence that when its causal etiology is framed biomedically both stigmatization and social exclusion increase $[15,16]$. At the same time, both for Tourette's as for diagnoses of mental disorder in general, a strict dichotomy between psychosocial and biomedical explanations, and therefore also the dichotomy in their effects, is now becoming obsolete. Indeed, for Tourette's specifically, scientific evidence points to an entanglement of biological and psychosocial factors with respect to expressing specific tics in behavior, for instance the evidence for the epigenetic nature of Tourette's given by Pagliaroli and colleagues [17]. Therefore, it could be enlightening to extend the exculpation-stigma literature towards Tourette's and further investigate empirically the dilemma between access to exculpation and stigma-mediated social exclusion (the mixed blessings or double-edged sword relations mentioned above) that seems at odds with both existing psychological and philosophical frameworks. Specifically, we were inspired by Strawson's ethical framework [18] where taking the 'objective attitude' towards atypical behavior leads to a form of exculpation, called 'exemption'. Whilst, at the face of it, such an objective attitude can inspire benevolent feelings (such as pity) toward those who are deemed categorically different - for instance on account of a deficit in their neurology - it comes at a significant interpersonal cost. Indeed, as noted by Strawson, the objective attitude is profoundly incompatible with 'the normal range' of human emotions (including for instance friendship and love). In the empirical literature there is ambivalence with respect to social distance: despite clear signs of stigmatization, it is not directly measured in classical social distance constructs [2]. Therefore, we wanted to explicitly revisit the measuring of social distance in a novel experimental design related to the specific case of Tourette Syndrome.

In the present study we aimed to investigate how blame for behavior common to Tourette's was related to a perception that these behaviors were generated by a biological, rather than a psychogenic cause. We further sought to investigate whether individuals expressed a greater desire for social distance when such behaviors were attributed to a psychosocial cause rather than to a psychogenic cause. Finally, we study if the more nuanced explanation, that entangles biological and psychosocial elements, impacts these measures.

\section{Methods}

\section{Recruitment}

The study was designed and executed using the online survey platform Qualtrics. We recruited 221 paid participants via the online participant recruitment service Prolific. Two eligibility criteria were applied. First, recruitment was limited to participants with English as their mother tongue given the study was entirely designed in English. Second, recruitment was limited to participants aged between 19 to 45 years to ensure all participants could imagine themselves in an environment of adult education. Ethical approval was obtained through the University ethical committee (approval number SHW_19_65 dd. 13/12/2019). A pre-registration of the study was done via the Open Science Framework (OSF) [19].

\section{Procedure}

The survey presented participants with a sequence of vignettes described in detail below with reference to Fig. 1. In an introductory vignette participants were asked to picture themselves in an adult education setting to align experiences across participants and also to anchor them in a situation that they could vividly imagine. The vignette read as follows:

"Picture yourself in a classroom for education to adults. This might be in university, college, corporate training or the like, whatever you can most vividly imagine."

After this introductory vignette, all participants were presented with three consecutive vignettes describing behavior that could disrupt an ongoing lecture. For each of the vignettes, participants were asked whether 


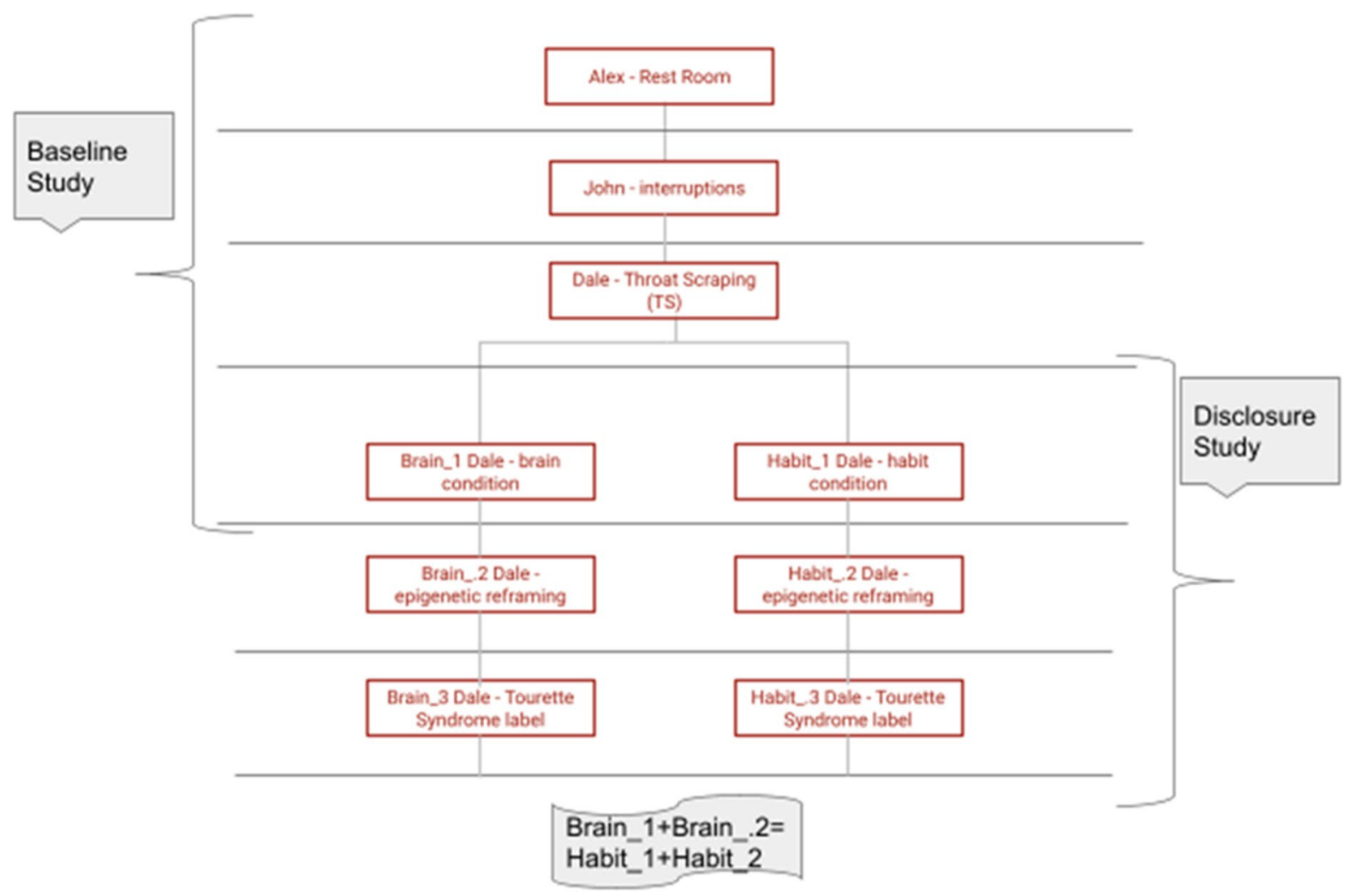

Fig. 1 Survey Flow

the person so behaving was to blame for their behavior on an ordinal scale with five response levels ranging from "Definitely not" (1) to "Definitely yes" (5). They were also asked to rate the extent to which they thought the behavior was caused by biological or by psychological factors on a 100-point analogue scale (0 fully biological; 100 fully psychological). We used an ordinal scale for blame so as to align with the experiments reviewed in the meta-review by Haslam and Kvaale [2]. We used an analogue sliding scale for the biological and psychological factors to allow participants to indicate the degree to which the behavior was seen as more or less the one or the other (see the Discussion and Limitations sections).

The three vignettes read as follows:

"Alex is one of your classmates. He regularly runs out of class to the restroom. The teacher asks him to stop behaving this way as it disrupts the class."

"John is one of your classmates. He regularly voices disagreement with the teacher. The teacher asks him to stop behaving this way as it disrupts the class."

"Dale is one of your classmates. He regularly scrapes his throat as if to say 'Ahum'. The teacher asks him to stop behaving this way as it disrupts the class."

The goal of these vignettes was to establish a baseline level of blame attributed for each of these behaviors and to gauge potential correlation between blame and the more neurological or psychological explanations for behavior. We selected the 'throat-scraping' tic as a phonic tic common to people diagnosed with Tourette Syndrome, and as such minimally necessary for diagnosis, whilst at the same time minimally stereotypical (see Limitations section for a discussion).

In order to understand the spontaneous reaction to behavior representative for Tourette's we introduce - contrary to the vignette studies reviewed in above mentioned reviews - this behavior, without linking it to the diagnostic label of Tourette Syndrome or to some specific etiological explanation. This allows us 
to baseline the variable 'Blame' for different types of disruptive behavior as well as correlate this variable to spontaneous attribution of whether the cause of such behavior is attributed rather to neurological or psychogenic causes.

After this baseline, the participants were randomly distributed over one of two conditions. In these conditions, Dale then took two alternate disclosure strategies in order to explain his behavior. The first was the 'Brain condition'. In this strategy, Dale started out explaining his behavior as flowing from him having a neurological condition. The second strategy was the 'Habit condition' in which Dale explained his behavior as having been left with bad habits based on his upbringing. These conditions mirror the "Dale"/"Sarah" vignettes of the thought experiment by Schroeder [13] described above, as well as nature (biological) versus nurture (psychogenic) accounts reviewed by Kushner in his historical account of Tourette's [4].

The vignettes read as follows:

Brain_1: "During the break you see Dale talking to the teacher. When class resumes, the teacher gives the floor to Dale, who explains that his throat-scraping is a tic flowing from how his brain works. He says it is more difficult to control when he is stressed."

Habit_1: "During the break you see Dale talking to the teacher. When class resumes, the teacher gives the floor to Dale, who explains that his throat-scraping is a habit flowing from how he was raised. He says it is more difficult to control when he is stressed."

After having been presented with these vignettes, we asked the participants in the respective conditions' context to revisit their blame attributions, after which they were presented with two questions related to social distance. The first was a measure we devised ourselves. In this we asked them how many friends they thought Dale had compared to an average individual. Answers were recorded on an ordinal scale ranging from "much less" (1) to "much more" (5). The second was a social distance construct regularly used in this type of studies. For our study, we reused the phrasing, and the scoring rules, of a recent autism disclosure study by White et al. [20], see Appendix 1 for direct reference. Such a multi-item social distance construct is traditionally used in the previous studies as reviewed by Haslam and Kvaale [2]. As they note, these traditional social distance constructs lead to inconclusive results in their meta-analysis.. For this reason we introduced our first alternative single-item question, to allow us to better discriminate participant responses across the conditions.

Starting from the baseline information above, we can compare the evolution of blame and desire for social distance in two conditions: one, the brain condition, where the behavior representative of Tourette Syndrome is explained as explicitly neurological and another, the habit condition, in which it is explained as explicitly psychosocial. In this first disclosure step, we do this without mentioning diagnostic labels in order to avoid introducing specific prejudices participants may have with respect to Tourette's.

In each of the conditions, a subsequent vignette was presented in which Dale explains his condition in more detail. This explanation was designed to align with views of Tourette Syndrome that suggest that psychosocial and biological factors co-contribute (an 'epigenetic' approach) to such behavior. Participants were asked to respond to the same set of questions as per the previous vignette. In both the brain and habit conditions this second vignette read as follows:

Brain_2/Habit_2: "You wonder about Dale and decide to ask him for details after class. He explains his doctor told him that recent research in a field called 'epigenetics' shows his behavior flows from an interplay of neurological and environmental factors. This explains there sometimes is an overlap between tics and what is commonly considered socially inappropriate habits."

In this way we checked how a more nuanced framing that entangles biological and psychosocial elements influences the evolution of attribution of blame and desire for social distance. In the vignettes this is referred to as an 'epigenetic' approach. This allowed us to explicitly study what effect more nuanced, less dichotomous, explanations have and if they help us to transcend the blame-stigma 'double-edged sword' or 'mixed blessings' dilemma.

In a naturalistic setting, explaining one's behavior would include, eventually, disclosing one's specific diagnostic label. For this reason we introduced in each of the conditions a third and last vignette. In this vignette Dale explicitly labeled his condition as 'Tourette Syndrome'. Again, we asked the participants to 
answer the same questions. The vignette read as follows in both conditions:

Brain_3/Habit_3: “In a next discussion Dale tells you he is diagnosed with Tourette Syndrome. He does not like to mention this straight away because it sometimes elicits one-sided reactions."

Because, as previously stated, several studies show ambivalent or insignificant differences on social distance constructs we asked the participants to answer how they felt about the survey, using a free text input (this option was again given after the debriefing statement).

"You almost finished the study. At this point, please share (this is completely optional) some of your thoughts with respect to the study. If you do, please limit this to 5-10 lines maximum and be sure not to provide any information breaking your anonymity."

At the end of the survey demographic information as to age, gender or direct acquaintance with people with a psychiatric, neurodevelopmental or very specifically Tourette Syndrome diagnosis was collected (either whether participants themselves fell in these categories or whether they knew someone who did, either as family or as friend). Analysis of this part of the data is outside of the scope of this paper.

\section{Results}

Our hypotheses and predictions on the different variables have been logged in our $\mathrm{OSF}^{4}$ pre-registration [19], both our raw data obtained as well as our analysis via SPSS have been logged there for reference too.

As to the first three vignettes. First, there was no significant difference in blame attribution between the Dale throat-scraping vignette (Mean 2.76, SD 0.967) and the Alex bathroom vignette (Mean 2.70, SD 0.992) as per a paired sample $t$-test $(t=-0.752, d f=220$, $\mathrm{p}<0.453$ ) (see further in Limitations). Second, a paired

\footnotetext{
4 The OSF data were not shared during the blind peer review process and as such were not reviewed by the anonymous reviewers (in line with review processes of non-pre-registered manuscripts).
}

sample t-test showed that the John interruption vignette did have significantly higher blame attribution (Mean 3.83 , SD 0.966) with respect to both the Alex and Dale vignettes (respectively: $\mathrm{t}=-12.030, \mathrm{df}=220, \mathrm{p}<0.001$ and $\mathrm{t}=12.138$, $\mathrm{df}=220, \mathrm{p}<0.001$ ). Third, responses to the Dale vignette trended toward higher blame attribution.

A next question across these three baseline vignettes (as preregistered), was to check whether higher blame attribution correlates with a spontaneously more psychological explanation of behavior by the participants. This was indeed the case. Figure 2 gives a statistical illustration of this result related to the Alex vignette. The mean and the $95 \% \mathrm{CI}$ on psychological explanation of behavior by participants were consistently higher for higher blame attribution. The graphs related to the two other vignettes exhibited the same pattern, clearly showing that the more blame is attributed by respondents, the higher the associated behavior is attributed to psychological causes. And vice versa, the less blame was attributed the more exclusively that type of behavior is attributed to biological causes.

Next, we compared blame attribution in the baseline Dale vignette with the blame attribution in the two conditions, following the presentation of the 'Brain_1' and 'Habit_1' vignettes.

Both conditions showed significant differences in blame attribution compared to the baseline. Comparing within the experimental group in the neurological condition showed a marked decrease of blame attribution after explaining Dale's behavior in neurological terms in the Brain_1 vignette (Mean 1,3, SD 0.549) when compared to that in the baseline Dale vignette (Mean 2,67, SD 0.937). The difference was highly significant according to a paired sample t-test $(\mathrm{t}=15.545, \mathrm{df}=110, \mathrm{p}<0.001)$. An unanticipated result was that blame was also significantly reduced in the experimental group in the psychosocial condition. After Dale's behavior was explained in a psychosocial way in the Habit_1 vignette blame attribution decreased (Mean 1,85, SD 0.826) compared to the one in the baseline Dale vignette (respectively Mean 2.86, SD 0.991). This difference was also significant according to a paired sample $\mathrm{t}$-test $(\mathrm{t}=12.964$, $\mathrm{df}=109, \mathrm{p}<0.001)$.

With respect to this element of our results, we finally note that the difference in blame reduction in the neurological and psychosocial conditions was 


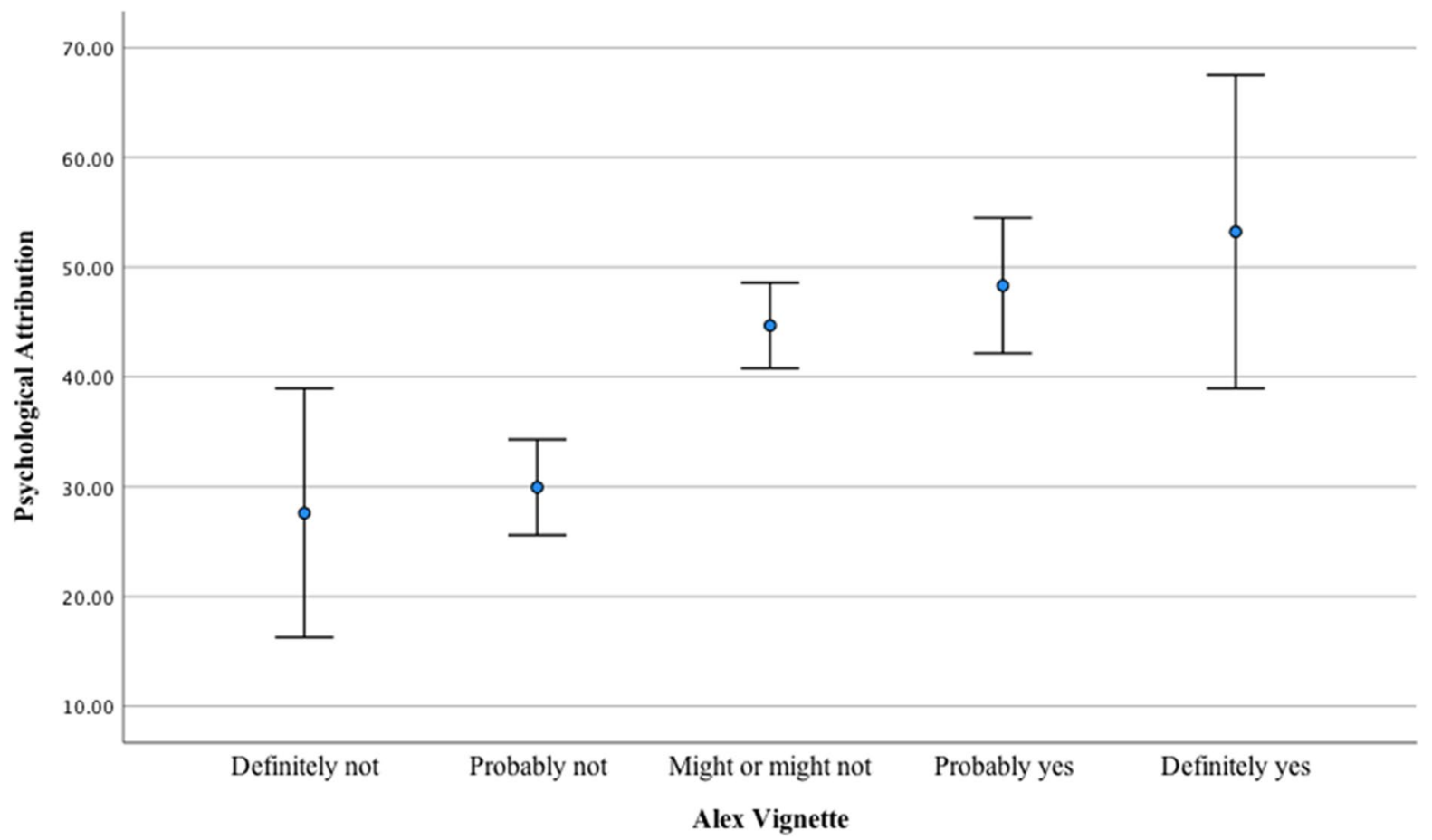

Fig. 2 Mean and 95\% CI psychological attribution as function of blame attribution

itself significant, as can be seen from non-overlapping Confidence Intervals on the difference between baseline blame attribution and blame attribution in the two conditions (Mean Diff 1.369/1.018, CI $=95 \%$ 1.194-1.545/0.863-1.174 neurological and psychosocial conditions respectively).

To conclude, we looked at the effects of introducing the second and third vignettes in each of the conditions. We started by analysing the blame attribution as it progresses with introduction of subsequent vignettes and then finalized statistical reports in doing the same with our two social distance measures.

As shown in Fig. 3, blame attribution in the two experimental groups responded differently to the introduction of the follow-on vignettes. In the brain condition, introduction of epigenetic information in the Brain_2 vignette did not affect blame attribution. Introduction of this same epigenetic information in the Habit_2 vignette did significantly reduce attribution of blame, in the direction of the blame attribution in the neurological condition. Explicit introduction of the Tourette diagnosis in the Brain_3 and Habit_3 vignettes resulted in further decrease of blame in both conditions. In fact, blame attribution across these two conditions converged after the introduction of the diagnostic label. We note that the total information received by all of the participants, as from the second vignette in either condition, was the same but presented in a different order.

In summary: whereas epigenetic framing did not impact in the brain condition, it did lead to less blame attribution in the habit condition. Introduction of the Tourette label resulted in a final change in both conditions: blame attribution converged. This was irrespective of whether respondents started out with a neurological or a psychosocial explanation.

With respect to the social distance measures: neither of the two measures were significantly different between conditions nor were they responsive to introduction of additional information within the conditions. For sake of clarity of exposition, we show this result for the interested reader in Appendix 3 (Figs. 4 and 5).

As mentioned in the Methods section, we also wanted to gauge the drivers for answering in a certain way, specifically with respect to the social distance measures given the inconclusive results reported in the review of Haslam and Kvaale [2] and replicated here. A free text field (with a substantive answer to the open question at the end of the survey) was filled out by 70 out of 221 
Fig. 3 Blame attribution mean and $95 \%$ CI per time point and condition

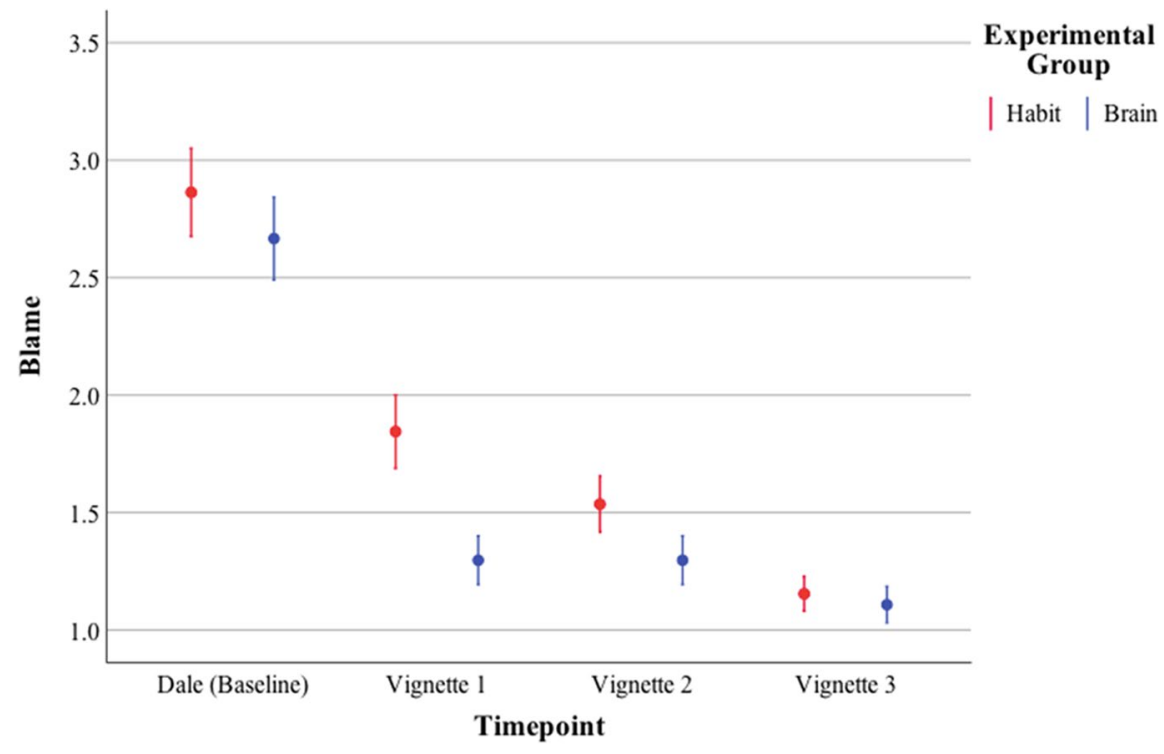

respondents (this is after discounting 18 answers related to the mechanics of the survey). This represents about $30 \%$ of respondents who gave some substantive qualitative feedback. Distribution of this feedback over participant gender and age was not remarkably different to overall participants' age and gender distribution reported higher. The amount of answers in either condition were about equal i.e. around 35 answers per condition. Most answers were short (10-30 words) with some exceptions (50-100 words). For clarity of exposition, we come back to the content and some samples of this feedback in the discussion (full data set is available from the OSF register).

\section{Discussion}

The results of our study have confirmed the pre-registered hypothesis (see OSF entry) that blame attribution covaries positively with psychological explanation of the disruptive behavior and negatively with biological explanation of that behavior. Likewise, they have confirmed that a neurological explanation lowers blame attribution more than a psychological explanation. This largely replicates findings reported in review studies [2, 3] in the case of Tourette Syndrome. An unanticipated result of our study is that, although a neurological explanation reduces blame attribution the most, psychological explanations also reduces blame attribution significantly with respect to the baseline level. At the same time, and as predicted, introducing an epigenetic explanation - in which biological and psychological factors were explicitly entangled - reduced blame attribution in the context of a prior psychological explanation but left it unchanged in the context of a prior biological explanation. Again as predicted, introduction of a 'Tourette Syndrome' label is associated with the lowest blame attribution, independent of previous explanations of the behavior. Finally, we did not confirm our hypothesis on the relative difference of social distance with respect to a psychological, neurological or epigenetic explanation in the vignettes. At the same time, this finding is in line with the conclusions of the meta-review by Haslam and Kvaale [2]. Our design for this reason allowed participants to share their reasoning via free text responses, giving us some qualitative data as a background to understand the rationale for their reactions.

We discuss the last four nuances brought to light by our study in more detail below.

First, whereas both in philosophy [13] as in the previous experimental paradigms [2, 3] biological and psychosocial explanations are treated as rival explanations, ${ }^{5}$ an unanticipated result of our study is

\footnotetext{
5 An anonymous reviewer correctly remarked that it would not be reasonable to portray all philosophical or psychological theorizing as committed to biological and psychosocial etiologies as rival accounts. However, this is not what we want to convey. We merely point to the important fact that they are sometimes so portrayed, and crucially so in the philosophical and experimental psychology literature that forms a starting point for our study. We want to explicitly recognize the nuances introduced by Haslam, Lebowitz and their colleagues, see for instance the last comment of this paragraph.
} 
that they rather work with different force but in the same direction. In our study, both explanations result in significant decrease of blame attribution albeit that the neurological explanation, in line with previous experimental results, has a more pronounced impact. This is all the more relevant since recent scientific evidence [17] for Tourette's suggests that it should not be considered as a phenomenon that arises only as a result of an individual's neurobiology. Based on this result it may be misleading to study a condition via the reactions to two contrast cases of biological and psychosocial framing respectively. Indeed, in so doing one would by design miss out a common effect, at least with respect to blame attributions, which is present in both. This may then lead to missing one possible way out of the mixed blessing or doubleedged sword binds, one that is already suggested by Lebowitz and Apelbaum [3]: an 'epigenetic' explanation of the behavior. ${ }^{6}$

Second, when one explicitly introduces such an epigenetic explanation we find that, on a psychosocial background, it clearly decreases blame attribution. How to interpret this result? In psychology, attribution theory [1] associates exculpation to control. The less control an agent has over certain aspects of their actions, the less they are blamed for them. A similar reasoning is given in some philosophical literature, for example Schroeder [13] uses Tourette Syndrome specifically to show how a deficit of inhibition on

\footnotetext{
${ }^{6}$ We are grateful to an anonymous reviewer for pointing out that our choice to present participants with a dichotomous continuous scale between fully biological and fully psychological explanations forces a negative relationship on these two variables. We will discuss this at length in the Limitations section, but we want to stress here that epigenetic explanations are in line with such a dichotomous continuous scale given a number of phenomena such as height are naturally seen as less under the influence of psychology, and others, such as feelings of anxiety, as more under such influence. Naturally, as also evident from results of our survey, participants will associate some phenomena as more to one or the other end of the scale. The dichotomy between psychology and biology can be seen as an idealization of such dynamics. Our results with the dichotomous continuous scale then support the idea that it is this idealization which leads to the mixed blessing or doubleedged sword double binds as they capture the underlying relation with blame but do so at the expense of obfuscating the nuance of epigenetic entanglement of these two complementary perspectives (psychosocial and biological) on the behavioral phenomena.
}

account of some neurological deficit would be a sufficient intuitive reason for not being blameworthy. ${ }^{7}$ What our results show is that absence of control because of putative neurological impairment is not the only dimension or criterion in play here. This points to more elaborate frameworks of moral responsibility in which the agent's intentions or 'Quality of Will' [21] also play a significant role. The framework proposed by Strawson [18] that inspired this study is a leading 'Quality of Will' approach. According to this approach there are two ways of gaining access to exculpation:

1. the behavior of another is considered with the 'participant attitude', where, as in our normal everyday practice, we excuse the other based on whether they showed us goodwill in trying to counteract various factors that might have compelled them to act in ways we deem inappropriate.

2. atypical behavior is considered to be determined by some categorical difference explaining why the other was completely determined to act in an inappropriate way. In this case we view them with the 'objective attitude'. The excuse we give then is an 'exemption', a standing excuse to act in certain ways because of an inability to act otherwise.

What our results show is that it is not necessary to gain access to exculpation via assumptions of a categorical difference anchored in a neurological deficit. A more nuanced approach to Tourette's is possible where the behavior is explained, like in our everyday responsibility practices, as an outcome of an entanglement of biological and psychosocial factors which still admit the person diagnosed to express their 'Quality

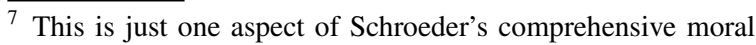
responsibility theory which is not merely a control theory as might appear by focusing only on the specific example of Tourette's. Arpaly and Schroeder [14] in fact develop a more comprehensive (non-Strawsonian) Quality of Will-theory where gradations of blameworthiness are explained as a confluence of factors (for instance in the case of addiction). That said, the Tourette Syndrime example shows they default to a control theory of moral responsibility in the case of 'neurological deficits', as the actions flowing from them do not come from (intrinsic) desires. At least in the Tourettic case our results contradict such a conclusive role of neurology as opposed to psychosocial factors in matters of 'psychopathological' moral responsibility.
} 
of Will'. Although these two ways of gaining access to exculpation would not be differentiated in quantitative results, they will make a substantive qualitative difference toward people diagnosed with Tourette's. Where the 'objective attitude' might be a shortcut to exculpation, it also sets a person seen in this way categorically apart from other human beings, in this case because of a deficit in their brain. As Strawson [18] notes this might be done for benevolent reasons and be compatible with positive emotions towards them but it is not compatible with 'the full range' of human emotions such as love, friendship and the like. It would therefore be intrinsically tied to some level of dehumanization of the Tourettic individual in accordance with the mixed blessing [2] or double-edged sword [3] dilemmas accompanying such 'standing' exculpation, or exemption. If, as our results indicate, people diagnosed with Tourette Syndrome can gain access to exculpation in Tourette Syndrome via the 'participant attitude' based on a more 'epigenetic' reading of the condition, then this risk of dehumanization can be avoided. As we will elaborate below with reference to social distance, such more nuanced reading then might allow to avoid stigma altogether without breaking access to a full range of empathy.

A third nuance relates to introducing the Tourette label explicitly. This leads to a decrease of blame attribution in both conditions and this at the lowest values recorded in our survey. ${ }^{8}$ We suspect that preconceptions around such labels, distinctly neurological for Tourette's at this point in time [4], effectively block out any additional information given. For this reason we believe disclosing labels up front in a survey, or in fact in actual disclosure practice-see White et al. [20]—-leads participants to answer in a 'socially desirable' way, thereby obfuscating how they would in fact react in the naturalistic settings where people diagnosed may opt not to disclose the label given the potential stigma attached to it.

This brings us to the fourth and final nuance: the social distance and stigma elements in our study. We have not been able to bring out a worsening of social distance in the responses to the vignettes, despite

\footnotetext{
$\overline{8}$ We are grateful to an anonymous reviewer for pointing out that the very low values for blame attribution at this stage may be indicative of a 'floor effect' within the ordinal 5-point scale used in out survey. We come back to this in the Limitations section.
}

introducing, alongside the traditional social distance construct, a new variable on estimating the amount of friends with respect to average. This aligns with the review of [3] where they suggest that social distance is to be considered as a downstream element where 'countervailing tendencies' are neutralized. They identify the possibility that there may be an upstream issue in empathy that is obfuscated in quantitative measures of social distance. Stigma associated with some condition would then be counterbalanced by feelings of pity for the people with these conditions and benevolent desires for their social inclusion despite their behavioral difference. This connects to the conclusion of Haslam and Kvaale [2] who do find, regardless of the inconclusive results on social distance, persistent components of stigma related to biomedical explanation. These elements are: prognosis, unpredictability as well as perceived dangerousness, all of these are connected to the ways in which people tend to be treated in real-life situations. Coming back to our discussion of Strawson's framework mentioned: both 'objective' and 'participant attitude' towards another are characterized by feelings of rapprochement. However, in the one case these are feelings of pity whereas in the other they are feelings of empathy which makes a crucial difference in actual practice. Indeed, helping behavior stemming from pity is often characterized as being a stigmatizing micro-aggression by disabled people [22] showing there is a real difference in (perceived) quality of engagement even if (or precisely because) the engagement itself is explained from benevolent motives. It therefore matters if the desire for social inclusion is motivated by a genuine desire to allow participation or stems from objectifying the other as 'in need' of 'help'. Even if social distance constructs cannot bring out this difference in a direct, measurable, way, the latter may well come with a stigmatizing attitude that is all the more frustrating as it defies direct measurement, something which is well expressed by the phrases of double-edged sword or mixed blessing with which we started this paper.

In fact, the qualitative data in response to our opentext feedback questions corroborate the assessment in these reviews of a double-edged sword or mixed blessing in case of static, neurobiological explanations. A closer look at these responses reveals that participants seem aware of such a disclosure dilemma on the part of people diagnosed with Tourette's regardless of the 
exculpatory potential of an explanatory framing. Many of our participants spontaneously bring up issues of social exclusion and stigma in their open-text feedback. Most telling were the comments that expressed an 'ought'. They suggest why it is difficult to measure social exclusion directly, as people want to give the socially desirable inclusive answer. Two examples are: "I don't think Dale should be treated differently because of his condition." (habit) and "I have a firm belief that everyone no matter what their physical or mental issues are should be treated the same as everyone else" (brain). Also prominent were the comments that indicated that the survey had made respondents reflect on their own attitudes. They suggest that regardless of the answers to the vignettes, participants do acutely feel there is a need for 'psychoeducation' to avoid potential stigma or exclusion. Two examples are:

"This made me reflect on the stigmas around mental health" (brain) and "This is a very interesting study as I do think the more people learn and understand about others and why others are 'acting' the way that they do, people then become more open and kinder to them (at least I would hope so)." (habit). Most troubling for an experimental survey method in this area are comments that suggest the motivation in answering in one way or the other depends on participant context rather than on (already artificial) vignette context. In and of itself this already suggests that quantitative studies like this one are to be complemented with qualitative studies querying values and motivations.

All in all, in these remarks it becomes apparent that even if social exclusion does not come out in the quantitative results, it may well be prominent in the way participants think about the presented case. In other words, they do see the the dilemma suggested by the 'double-edged sword' or 'mixed blessing' phrases in the review studies referenced above. This also explains the origin of the pressure people diagnosed with Tourette's feel to avoid tics. By 'passing' as normal, they do not have to explain their condition and disclose their diagnostic label, thereby avoiding the issue. This pressure is clearly brought out in empirical study of stigma in Tourette Syndrome $[15,16]$ as well as evident in the disclosure studies related to autism [20].

\section{Conclusion}

Our results indicate that being given a neurological explanation for a behaviour has as a result that blame attribution is decreased. Nevertheless, this should not blind us from long term stigmatizing effects of reducing the condition to a genetic or neurological deficit as indicated in the review studies [2,3]. Indeed, the remarks our participants filled in in the text boxes indicate that our participants do feel this 'doubleedged sword' or 'mixed blessing' tension between simplicity of explanation and the risk of social exclusion through stigma. In this sense, our results support the suggestion of [3] that social distance constructs might be incapable of bringing out this tension because of 'countervailing tendencies' operative in answering such items.

Our results indicate that there is potential for avoiding an either/or situation with respect to biological or psychosocial framing of conditions. Indeed, contrary to existing philosophical [13] and psychological frameworks [1] both types of explanation result in a decrease of blame attribution. Leaving a dichotomy between biological (nature) and psychosocial (nurture) explanations, we find that mixing them in a dynamic epigenetic explanation where biological and psychosocial elements are entangled, does not increase blame attribution. Exploring such more nuanced explanatory frameworks is not only more compatible with recent scientific results [17], it may provide a way for dissolving tensions expressed by the 'double-edged sword' and 'mixed blessing' phrases, tensions which are arguably at the core of the lived experience of Tourette's. That such an alternative for escaping stigma is crucial, is evident from the fact that Quality of Life issues in Tourette's are to a large extent associated with stigma, for instance via 'feelings of isolation, loneliness, and experiences of bullying" [12].

We believe, therefore, that a more nuanced approach to conceptualizing the neurology of Tourette Syndrome (and by extension of other mental 'conditions') needs to be explored in tandem with a more elaborate moral responsibility framework. According to the framework of Strawson [18], initially inspiring the present study, such a nuanced approach could allow for exculpation 
without running the risk of stigma via dehumanization [2]. Indeed, oversimplifying neurological explanations according to a simple nature/nurture dichotomy only leaves room for seeing the condition as a 'static deficit' and therefore viewing the people so diagnosed with an 'objective attitude'. But admitting more dynamic epigenetic conceptualizations - see also Lebowitz and Apelbaum [3]—would, in principle, admit viewing them with a 'participant attitude' allowing them exculpation based on their neurological difference without thereby labeling them as categorically different on a basic aspect of our social and moral functioning. Exploring this option cannot be done, in our view and based on our study, merely by quantitative study alone as the element of pity mentioned by Lebowitz and Apelbaum [3] and Strawson [23] might obfuscate underlying tendencies of stigma-mediated social exclusion. Alongside more nuanced ways of conceptualizing Tourette Syndrome, we suggest a thorough qualitative study of how the people diagnosed with Tourette Syndrome and their direct contacts come to terms with the condition.

\section{Limitations}

'Common' tics like throat-scraping are initially interpreted as being caused by something biological. They therefore attract less blame than explicitly socially inappropriate behavior. It is difficult to "find the balance' in presenting Tourette Syndrome behavior in one vignette. We have opted for the mild 'throat-scraping' tic in order not to contribute to a stereotyping that hurts people diagnosed. However, results might have been more pronounced when more inappropriate behavior was chosen within the baseline condition.

In the baseline part of our study, we only established the baseline for blame attribution, but not for the 'social distance' variables. We can therefore only say that these two etiological explanations have no measurable different impact on social distance. It remains open, and interesting in terms of our speculation, whether or not they would both have a similar impact on social distance.

Further on the baseline part of our study, we are grateful to an anonymous reviewer to put pressure on our choice for forcing participants to choose to pinpoint disruptive behavior to a dichotomous continuous scale between fully biological and fully psychological. Whilst we believe (see footnote 5) this choice is not only compatible with our 'epigenetic' conclusions but, in fact, supportive of them, we acknowledge that our design forces a negative relation between these two variables. If it turns out, as our anonymous reviewer believes, that this implicit assumption is not defensible then this part of our results is invalid. However, even in such an event (which we argue against in footnote 5), the rest of our discussion can, as the anonymous reviewer also points out, stand. That said, the heart of the matter, as rightly noted by the anonymous reviewer, is that "the fully biological and fully psychological beliefs need not be mutually exclusive, as the measure might imply. It is feasible a participant may believe that behavior is both fully biological and fully psychological, for example, rather than either one or the other. My own belief would be something along these lines. The perceived causal influence of biological factors need not, in my opinion, outweigh or come at the expense of social or psychological factors. If I had a biological tendency to be inattentive, this will in turn influence my interactions with the world and people around me, which in turn may have psychological implications (or vice versa...). As a result I would equally want to answer both 20 and 80 on this scale, and so might be forced to use the midpoint instead, which could equally be interpreted as **neither** psychological nor biological, which would not be my intention." This remark is, of course, in line with our 'epigenetic' conclusion and merits further investigation in its own right. Whilst we cannot address this in the scope of the current paper, there need not, in our view, be an incompatibility between biological and psychological factors being entangled in behavioral tendencies and an instance of behavior, like tics, being assessed, in the moment, of being the result of either more psychological or biological factors.

An anonymous reviewer pointed us to a statistical limitation of our method. We have relied on an ordinal scale ${ }^{9}$ for blame attribution in line with previous experimental paradigms such as reviewed by Haslam

\footnotetext{
9 We are well aware of the controversy surrounding the analysis of ordinal variables (see e.g., [23, 24]. However, in the present analyses we invoke the basic principle that the $t$ test is uniformly most powerful [25], meaning, that the t test is the most robust statistical test to discover whether or not there exist differences between experimental groups.
} 
and Kvaale [2]. Doing so however, may introduce 'floor effects' because our values for blame attribution approach, certainly in the third vignette, an, in our set-up, minimal value of 1 . At that time it becomes difficult to see to what extent decreases are due to changes in the vignette or due to participants' inability to express nuance on the lower end of the scale. It may even be the case that introducing more information leads to a 'floor effect' independent of the type of information (whether biological, psychological or epigenetic) introduced. We agree to these caveats (which are in line with our conclusion to not only rely on quantitative methods in these matters, see also below) but believe the result on epigenetic explanations as a possible way out of the 'mixed blessings' or 'double edged sword' double binds is, nevertheless, robust enough to be interesting.

As some of our participants pointed out, we have not phrased our responsibility question in a more prospective, forward looking, kind of way; for instance by asking whether or not the participants believed Dale or others should take measures to avoid situations of disruption. Based on discussions in the moral responsibility literature [21], it is of interest to study whether other ways to frame the vignettes or the blame or social distance variables might be better in uncovering quantitative differences related to exculpation and stigma. Whilst we hope this discussion inspires innovation in quantitative, experimental surveys in this area, we believe it sets limits to the extent in which such surveys can shed light on the complex phenomenon of stigma. A first glimpse of these limits are provided via the narrative data collected at the end of this survey. As our conclusion suggests, a closer qualitative study of the practice of dealing with Tourette Syndrome behavior, in all of its situational complexity, can reveal intricacies shielded by initially relying on the quantitative method. Final conclusions should therefore, in our opinion, not be made without exploring this qualitative detail. This speaks, in our opinion, to the limits of the quantitative methods in experimental philosophy when done with the purpose of coming to universal and definite conclusions on matters related to the full complexity of human relations [26].
That said, our interpretation of the qualitative data in the free text responses is based on only a subset of respondents. It is also very tentative compared to the methods which need to be applied in rigorous qualitative research. The narrative data seems in any case to be largely independent of whether it is presented in the brain or habit framing. It points to a need to investigate how in their everyday practice, people navigate tensions expressed by phrases like double-edged sword or mixed blessing. It would seem to be the case that in everyday practice people explore an inbetween territory that judges behavior and people in a more dynamic way. Exploring such dynamics apparently depends on the specific context of their own situation, their relation and life history. So even if our interpretation of the qualitative data is not definitive, we believe the requirement for in-depth qualitative research is well founded.

Acknowledgements We want to thank Laura Matthys for her invaluable help in designing the present study. We thank Emma Moormann for her helpful comments on earlier drafts of this paper. Finally, we stress that the present study design greatly benefited from multiple discussions within the NeuroEpigenEthics team.

Author Contributions JB: initial study design, interpretation and first draft of manuscript. JKK: interpretation of study results and reviews of manuscript. $\mathrm{KH}$ : initial study design and reviews of manuscript.

Funding JB/KH were supported by the NeuroEpigenEthics project which received funding from the European Research Council (ERC) under the European Union's Horizon 2020 research and innovation program (grant agreement No 804881).

Data Availability Everything available via public OSPF project: https://osf.io/usykt/.

Code Availability Not applicable.

\section{Declarations}

Ethics and Consent Ethical approval for this study was obtained prior to conducting it from the relevant ethical committee on social sciences of the University of Antwerp (reference: SHW_19_65). Both consent and debriefing were an integral part of the Qualtrics survey, all details contained in Bervoets and Hens (2020).

Conflicts of Interest Nothing to declare. 


\section{Appendix 1: Social Distance Questionnaire}

Participants are asked to rate their agreement with the following sentences about Dale and this on a five-point scale $(1=$ Strongly disagree, $2=$ Disagree, $3=$ Not sure, $4=$ Agree, 5=Strongly agree). Items 1, 4, 6 and 8 are reverse-scored. A higher score indicates desire for more social distance from the target.

1. I could be friends with Dale.

2. I don't want Dale to join my class.

3. If I saw Dale in the corridor I'd avoid him.

4. I'd be happy to spend time with Dale out of school.

5. I wouldn't introduce Dale to my friends.

6. I'd be happy to work on a group project with Dale.

7. I'd never go to Dale for help with a personal problem.

8. If Dale was in trouble I'd help him.

\section{Appendix 2: Debriefing Statement}

This study was designed to gauge your reaction to behavior typical for Tourette Syndrome ('Dale' case) and how you would react when somebody diagnosed with Tourette syndrome would explain this behavior.

Tourette Syndrome is often mischaracterized in society by associating it to the compulsive uttering of swear words (coprolalia). In fact, this behavior only affects about $15 \%$ of people diagnosed with Tourette Syndrome. The official diagnostic criteria (per DSM-5) are multiple motor tics and at least one vocal tic starting before 18 years. Vocal tics can for instance be sniffing, throat scraping or blowing raspberries. Behavior in the 'Dale' vignettes is therefore typically present in cases of diagnosis with Tourette Syndrome. It has also been validated as typical and non-stigmatizing by people diagnosed with Tourette Syndrome and by specialists working on Tourette Syndrome.

Please be aware Tourette Syndrome isn't a rare condition. It is currently estimated to affect around 1 in a 100 individuals. It is therefore not unlikely you have met (or indeed will meet) someone with Tourette's.
As to the causes of Tourette's, the current consensus is that it is neurobiological in nature. However, no specific genetic or neurological causes have as of yet been pinpointed. There is ongoing research into how someone's environment influences tics both in frequency and in content. This suggests that there may be at least some environmental factors impacting on the (controllability of) tics. In this sense the vignettes are in line with possible epigenetic explanations of Tourette Syndrome saying that tics flow from an interplay of biological and environmental factors. That said more research needs to be done before we can make any more definitive statements about this.

This survey is designed to see how people react to tics, how this drives the need for those diagnosed to disclose their condition and how the explanation given for their behavior (sometimes at a time they are not yet themselves aware of their diagnosis) impacts the way others see them. Whatever the result of this study and further scientific insights in Tourette Syndrome, what is clear is that the reactions of others have, via stress, a major impact on the urge to tic and the quality of life of those diagnosed. Your participation in this survey will therefore inform us one way or another how to potentially make life better for people with Tourette Syndrome.

\section{Appendix 3: Social Distance Measures}

Figure 3 gives the progression across the vignettes in the two conditions for the first social distance measure asking participants whether Dale had more or less friends than average (cfr Methods section: the higher this measure the higher the number of friends participants thought Dale would have, the measure can range between 1 and 5).

Figure 4 gives progression across the vignettes in the two conditions for the second social distance measure. In this case the measure as depicted is the average of the total social distance construct computed per Appendix 1 over the 8 constituent items. The higher this measure the higher the social distance that participants would take from Dale (cfr Methods Section, the measure can range between 1 and 5). 
Fig. 4 Mean and 95\% CI of 'Friends' measure per time point and condition

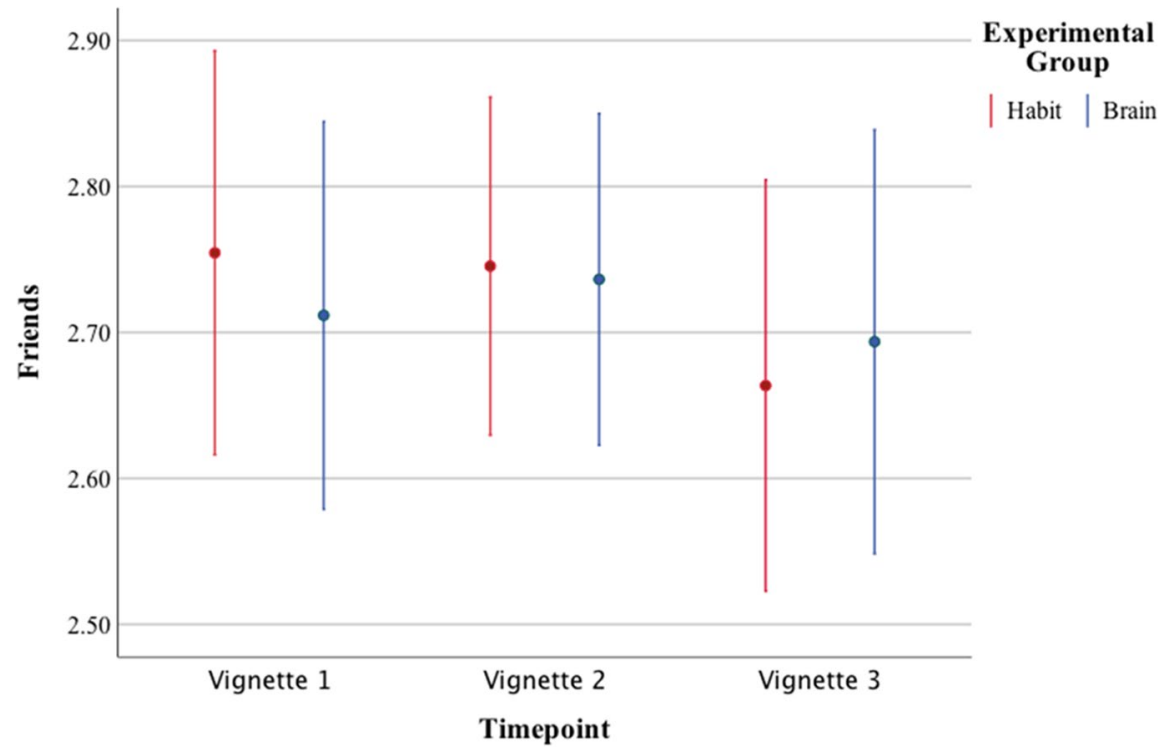

Fig. 5 Mean and 95\% CI of Social Distance Construct per timepoint and condition

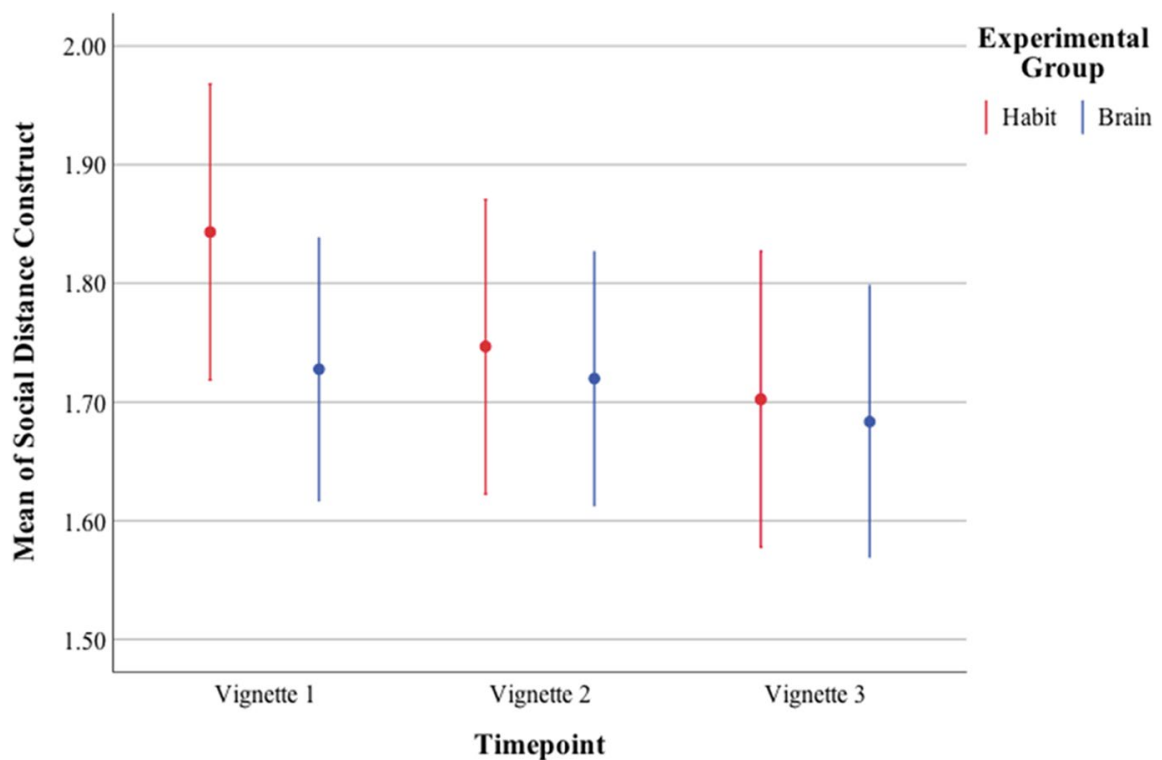

Open Access This article is licensed under a Creative Commons Attribution 4.0 International License, which permits use, sharing, adaptation, distribution and reproduction in any medium or format, as long as you give appropriate credit to the original author(s) and the source, provide a link to the Creative Commons licence, and indicate if changes were made. The images or other third party material in this article are included in the article's Creative Commons licence, unless indicated otherwise in a credit line to the material. If material is not included in the article's Creative Commons licence and your intended use is not permitted by statutory regulation or exceeds the permitted use, you will need to obtain permission directly from the copyright holder. To view a copy of this licence, visit http://creativecommons.org/licenses/by/4.0/.

\section{References}

1. Weiner, Bernard. 1985. An Attributional Theory of Motivation and Emotion. Springer Series in Social Psychology, Springer.

2. Haslam, Nick, and Erlend P. Kvaale. 2015. Biogenetic Explanations of Mental Disorder. Current Directions in Psychological Science.

3. Lebowitz, Matthew S., and Paul S. Appelbaum. 2019. Biomedical Explanations of Psychopathology and Their Implications for Attitudes and Beliefs About Mental Disorders. Annual Review of Clinical Psychology 15: $555-577$. 
4. Kushner, Howard I. 1999. A Cursing Brain? The Histories of Tourette Syndrome: The Histories of Tourette Syndrome. Harvard University Press.

5. American Psychiatric Association. 2013. Diagnostic and Statistical Manual of Mental Disorders (DSM-5®). American Psychiatric Pub.

6. Beljaars, Diana. 2019. Towards Compulsive Geographies. Transactions of the Institute of British Geographers.

7. Kim, Soyoung, Deanna J. Greene, Emily C. Bihun, Jonathan M. Koller, Jacqueline, M. Hampton, Haley Acevedo, Angela M. Reiersen, Bradley L. Schlaggar, and Kevin J. Black. 2019. Provisional Tic Disorder Is Not so Transient. Scientific Reports. https://doi.org/10.1038/s41598-019-40133-4.

8. Groth, Camilla, Liselotte Skov, Theis Lange, and Nanette M. Debes. 2019. Predictors of the Clinical Course of Tourette Syndrome: A Longitudinal Study. Journal of Child Neurology 34 (14): 913-21.

9. Ben-Ezra, M., S. Anavi-Goffer, E. Arditi, P. Ron, R.P. Atia, Y. Rate, and K. Kaniasty. 2017. Revisiting stigma: Exposure to Tourette in an ordinary setting increases stigmatization. Psychiatry Research 248: 95-97.

10. Robertson, Mary May, and Valsamma Eapen. 2014. Tourette's: Syndrome, Disorder or Spectrum? Classificatory Challenges and an Appraisal of the DSM Criteria. Asian Journal of Psychiatry 11 (October): 106-113.

11. Eddy, Clare M., and Andrea E. Cavanna. 2013. On Being Your Own Worst Enemy: 26, An Investigation of Socially Inappropriate Symptoms in Tourette Syndrome. Journal of Psychiatric Research.

12. Cox, J.H., A. Nahar, C. Termine, M. Agosti, U. Balottin, S. Seri, and A.E. Cavanna. 2019. Social stigma and selfperception in adolescents with tourette syndrome. Adolescent health, medicine and therapeutics 10: 75-82. https:// doi.org/10.2147/AHMT.S175765.

13. Schroeder, Timothy. 2005. Moral Responsibility and Tourette Syndrome. Philosophy and Phenomenological Research.

14. Arpaly, Nomy, and Timothy Schroeder. 2013. In Praise of Desire. Oxford University Press, Oxford. https://doi.org/ 10.1093/acprof:oso/9780199348169.001.0001.

15. Malli, Melina A., Rachel Forrester-Jones, and Glynis Murphy. 2016. Stigma in Youth with Tourette's Syndrome: A Systematic Review and Synthesis. European Child \& Adolescent Psychiatry 25 (2): 127-139.
16. Malli, Melina A, R Forrester-Jones, P Triantafyllopoulou. 2019. Tourette's Is a Lonely Place: an Interpretative Phenomenological Analysis of the Personal Experience and Identity of Adults with Tourette's Syndrome. Journal of Developmental and Physical Disabilities.

17. Pagliaroli, Luca, and Borbála Vető, Tamás Arányi, and Csaba Barta. 2016. From Genetics to Epigenetics: New Perspectives in Tourette Syndrome Research. Frontiers in Neuroscience 10 (July): 277.

18. Strawson, P.F. 2008. Freedom and Resentment and Other Essays. London: Routledge.

19. Bervoets, J., and Hens, K. (2021). Link Exculpation/Stigmatization Tourette Syndrome (NeuroEpigenEthics X-Phi 1). Retrieved from https://osf.io/usykt/.

20. White, R., M. Barreto, J. Harrington, S.K. Kapp, J. Hayes, and G. Russell. 2020. Is disclosing an autism spectrum disorder in school associated with reduced stigmatization? Autism 24 (3): 744-754.

21. Talbert, Matthew. 2019. Moral Responsibility. The Stanford Encyclopedia of Philosophy (Winter 2019 Edition), Edward N. Zalta (ed.). https://plato.stanford.edu/archives/win2019/entries/ moral-responsibility/.

22. Keller, R.M., and C.E. Galgay. 2010. Microaggressive experiences of people with disabilities. In Microaggressions and marginality: Manifestation, dynamics, and impact, ed. D.W. Sue, 241-267. John Wiley \& Sons Inc.

23. Kampen, J.K., and M. Swyngedouw. 2000. The ordinal controversy revisited. Quality \& Quantity 34 (1): 87-102.

24. Liddell, T.M., and J.K. Kruschke. 2018. Analyzing ordinal data with metric models: What could possibly go wrong? Journal of Experimental Social Psychology 79: 328-348.

25. Lehman, E.L. 1975. Non-parametrics: Statistical methods based on ranks. San Francisco: Holden-Day.

26. Fischer, E. 2018. Wittgensteinian 'therapy', experimental philosophy, and metaphilosophical naturalism. In K. Cahill and T. Raleigh (eds.): Wittgenstein and Naturalism (pp. 260-286). New York: Routledge.

Publisher's Note Springer Nature remains neutral with regard to jurisdictional claims in published maps and institutional affiliations. 